\title{
IMPACT OF TEC GRADIENTS AND HIGHER-ORDER IONOSPHERIC DISTURBANCES ON SPACEBORNE SINGLE-PASS SAR INTERFEROMETRY
}

\author{
G. Krieger, F. De Zan, P. Lopez Dekker, J-S. Kim, M. Rodriguez Cassola, A. Moreira
}

\author{
German Aerospace Center (DLR)
}

\begin{abstract}
This paper analyzes the impact of a spatially inhomogeneous ionosphere on spaceborne single-pass SAR interferometry. For this, linear TEC gradients and higher-order irregularities are considered. It is shown that TEC gradients as low as 0.01 TECU/km may already noticeably affect the accuracy of an L-band cross-track interferometer, causing, e.g., horizontal and vertical offsets in the order of 1-2 $\mathrm{m}$. Higher-order perturbations of the electron plasma lead to additional errors that vary nonlinearly with the length of the interferometric baseline. To predict these errors, we model the ionospheric irregularities as the product of a vertical profile and a secondorder stationary stochastic process with a 3-D power-law spectrum. The interferometric errors are then derived via a set of projection integrals that express the expected phase error variance as a function of the baseline length and the angular extent of the synthetic aperture. With this model, we show that the phase errors of an L-band single-pass SAR interferometer may reach several tens of degrees under medium turbulence conditions. Since these phase errors are highly correlated among neighboring resolution cells, they cannot be reduced by multi-looking, thereby posing a possible challenge for multiple baseline interferometry and SAR tomography.
\end{abstract}

Index Terms - SAR interferometry, ionosphere, TEC gradients, scintillation, irregularity spectrum, turbulence

\section{INTRODUCTION}

It has often been claimed that single-pass SAR interferometry is, up to a common range shift of both interferometric channels, not affected by the atmosphere. In [1], it has, however, been shown that the slightly different ray paths through a vertically stratified and horizontally homogeneous troposphere can cause notable differential phase and range offsets. Small differential tropospheric delays explain, for example, the systematic height errors of up to three meters that have been observed in the TanDEM-X mission [2]. Single-pass SAR interferometers operating at lower frequencies, like the planned Tandem-L and SAOCOM-CS missions, may furthermore be affected by differential ionospheric propagation effects. Since the ionosphere is a dispersive medium, one must here distinguish between differential range delay and interferometric phase advance, which cause opposite DEM offsets for SAR interferometry and radargrammetry. Such offsets have, together with additional intricacies from spectral shift, been analyzed in [1] for the case of a spatially homogeneous ionosphere, where it has been shown that

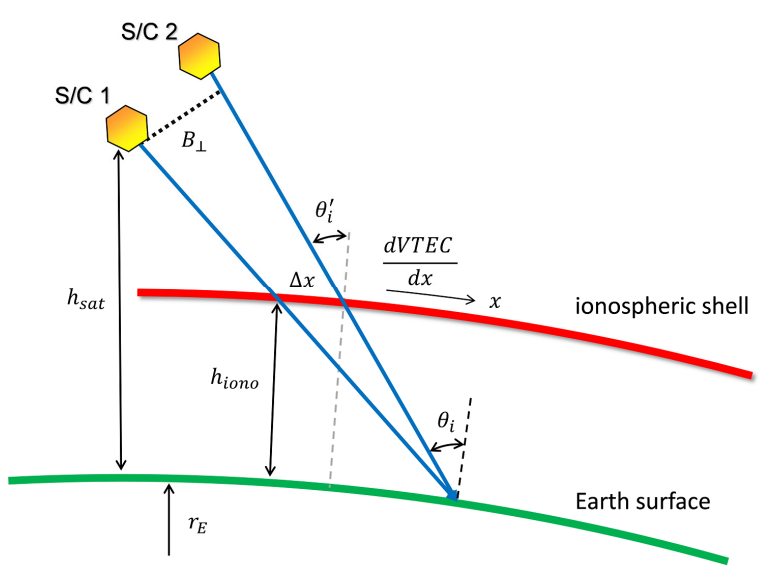

Figure 1: Geometry to analyze the impact of TEC gradients on single-pass cross-track interferometry.

height errors of several tens of meters must be expected at times of moderate to strong solar activity. In this paper, we extend these analyses to a space-variant ionosphere, where we consider in Section 2 linear slopes of the total electron content (TEC), and in Section 3 higher-order electron density perturbations. Due to space limitations, we restrict our discussion to differential effects that arise for the phase of a single-pass SAR interferometer. The impact of TEC variations on the focusing of single SAR images has already been treated in detail in the literature [3], [4], [5], [6].

\section{LINEAR TEC GRADIENTS}

We consider first a linear TEC gradient in the cross-track direction. Figure 1 illustrates how the rays from the two antennas intersect the ionosphere which is in this section modelled as a thin spherical shell layer. The distance $\Delta x$ separating the two intersection points between the rays and the ionospheric shell can be approximated as

$$
\Delta x \approx \frac{h_{\text {iono }}}{h_{\text {sat }} \cdot \cos \theta_{i}^{\prime}} \cdot B_{\perp}
$$

If the gradient of the (vertical) TEC in range direction $x$ is given by $\partial V T E C / \partial x$, we obtain for the slant TEC difference $\triangle S T E C$ between the two acquisitions

$$
\triangle S T E C \approx \frac{h_{\text {iono }}}{h_{\text {sat }} \cdot \cos ^{2} \theta_{i}^{\prime}} \cdot \frac{\partial V T E C}{\partial x} \cdot B_{\perp}
$$



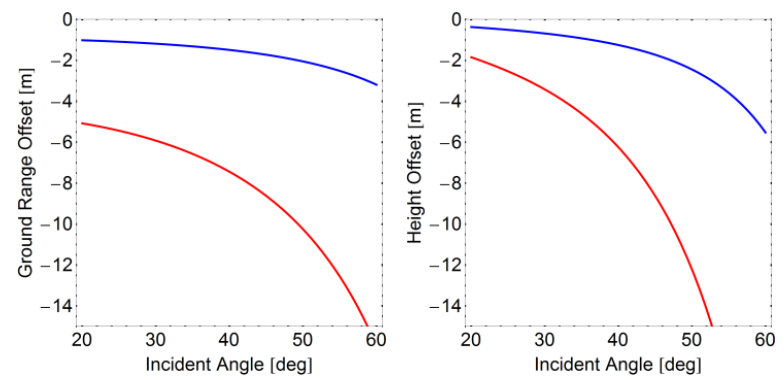

Figure 2: Horizontal (left) and vertical (right) offsets of the interferometric DEM caused by TEC gradients in the crosstrack direction. The red and blue curves show the predicted displacements of an L-band SAR interferometer for TEC gradients of $0.01 \mathrm{TECU} / \mathrm{km}$ and $0.05 \mathrm{TECU} / \mathrm{km}$, respectively. The height of the satellite and the ionospheric shell layer are $h_{\text {sat }}=700 \mathrm{~km}$ and $h_{\text {iono }}=350 \mathrm{~km}$, respectively.

The corresponding horizontal and vertical DEM offsets can then be derived in analogy to the derivations in [1] as

$$
\begin{aligned}
\Delta x_{\text {horizontal }}^{\text {gradiono }} & = \pm \frac{K}{f^{2}} \cdot \frac{\partial V T E C}{\partial x} \cdot \frac{h_{\text {iono }}}{h_{\text {sat }}} \cdot r_{\text {slant }} \cdot \frac{1}{\cos \theta_{i}^{\prime}} \\
\Delta x_{\text {vertical }}^{\text {gradiono }} & = \pm \frac{K}{f^{2}} \cdot \frac{\partial V T E C}{\partial x} \cdot \frac{h_{\text {iono }}}{h_{\text {sat }}} \cdot r_{\text {slant }} \cdot \frac{\tan \theta_{i}^{\prime}}{\cos \theta_{i}^{\prime}}
\end{aligned}
$$

where $f$ denotes the radar carrier frequency, $r_{\text {slant }}$ the slant range, and $K=40.28 \mathrm{~m}^{3} / \mathrm{s}^{2}$. The positive signs apply to radargrammetry and the negative signs to interferometry. Note that the DEM offsets are, as for the case of a constant TEC, independent of the baseline length. Figure 2 shows the predicted horizontal and vertical interferometric DEM offsets for an L-band interferometer operating at two different TEC gradients. It becomes clear that TEC gradients above $0.01 \mathrm{TECU} / \mathrm{km}$ can already lead to noticeable horizontal and vertical errors in the order of 1-2 meters that increase with increasing incident angles. Typical TEC gradients are characterized by a high degree of spatial and temporal variability, ranging from less than 0.01 $\mathrm{TECU} / \mathrm{km}$ up to $0.08 \mathrm{TECU} / \mathrm{km}$; under extreme conditions TEC gradients may reach values of $0.5 \mathrm{TECU} / \mathrm{km}$ [7], [8]. From this, it becomes clear that ionospheric effects should be taken into account when designing bistatic L-band SAR missions like Tandem-L or SAOCOM-CS.

A TEC gradient in azimuth will, to first order, add a linear phase ramp to each synthetic aperture. As a result, both SAR images, and hence also the DEM, will be shifted in azimuth. Assuming zero-Doppler geometry and no TEC variations in range, the two SAR image acquisitions will be affected by (almost) the same phase gradients, and therefore no additional height offsets beyond those of a constant ionosphere are expected. This applies even to the case of a bistatic acquisition with a non-vanishing along-track baseline, as long as the centroid of the common processed Doppler spectrum does not become too large. Joint azimuth and range gradients can, however, cause additional errors, e.g., due to mis-registration in azimuth.

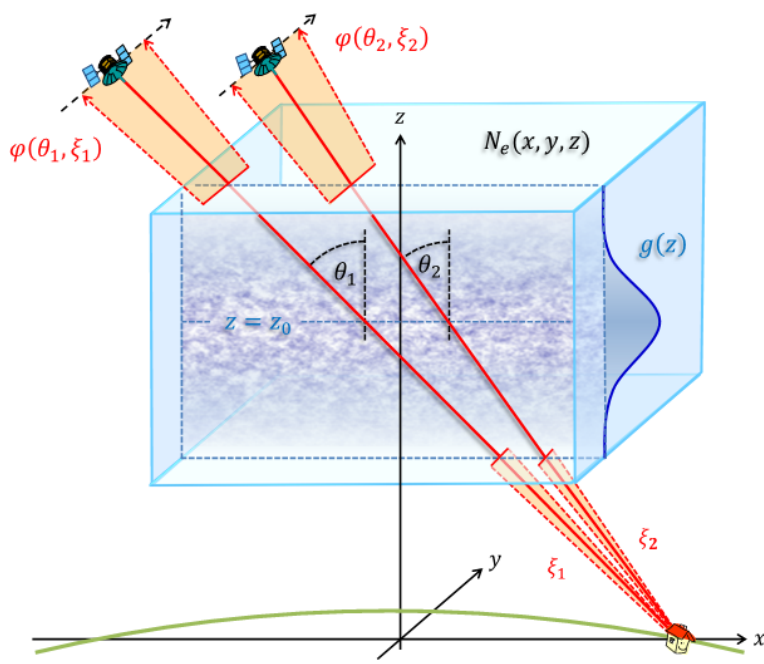

Figure 3: Geometric model describing the differential ionospheric phase advance of two rays emerging from a single point-like scatterer on the ground. The two rays pass different portions of the turbulent ionosphere which is characterized by the product of a deterministic vertical density profile $g(z)$ and a spatially homogeneous stochastic electron density distribution $N_{e}(x, y, z)$. The different incident angles at height $z_{0}$ are denoted by $\theta_{1}$ and $\theta_{2}$, while the angles $\xi_{1}$ and $\xi_{2}$ refer to the formation of the synthetic aperture.

\section{IONOSPHERIC TURBULENCE}

The equatorial, auroral and polar ionosphere are often characterized by notable ionospheric irregularities [9], [10]. To study the impact of such irregularities on single-pass SAR interferometry, we assume a so-called frozen ionosphere which exhibits only spatial but no temporal fluctuations of the electron density within the observation interval. Referring to Figure 3, we model the ionosphere as a locally flat slab with a vertical electron density profile $g(z)$. The local electron density irregularities are moreover described by a 3-D homogeneous stochastic process $N_{e}(x, y, z)$ that accounts for all deviations of the electron density from a mean background. Assuming that the ratio between the local electron density fluctuations and the electron background density is constant [11], we can express the actual 3-D electron density as the product of the vertical profile $g(z)$ and a realization of the homogeneous stochastic process $N_{e}(x, y, z)$. To proceed, we further assume that $N_{e}(x, y, z)$ is zero mean second-order stationary. The spatial statistics of $N_{e}(x, y, z)$ can therefore be described in terms of the translationally invariant 3-D autocorrelation function

$$
\begin{aligned}
C_{N_{e}}(\Delta x, \Delta y, \Delta z)= & \mathrm{E}\left[N_{e}(x, y, z)\right. \\
& \left.\cdot N_{e}(x+\Delta x, y+\Delta y, z+\Delta z)\right]
\end{aligned}
$$

where E[] denotes the expectation operator. In the ionospheric literature it is common praxis to use instead of the autocorrelation function $C_{N_{e}}(\Delta x, \Delta y, \Delta z)$ the 3-D electron density power spectrum $S_{N_{e}}\left(f_{x}, f_{y}, f_{z}\right)$ which is 
related to $C_{N_{e}}$ by a 3-D inverse Fourier transform [9]

$$
\begin{aligned}
& C_{N_{e}}(\Delta x, \Delta y, \Delta z)=\iiint_{-\infty}^{\infty} S_{N_{e}}\left(f_{x}, f_{y}, f_{z}\right) \cdot \\
& \exp \left(j 2 \pi\left(f_{x} \Delta x+f_{y} \Delta y+f_{z} \Delta z\right)\right) \cdot d f_{x} d f_{y} d f_{z}
\end{aligned}
$$

Based on this second-order stochastic model, we may now analyze the statistical dependencies of the phase fluctuations for two rays that penetrate the ionosphere as illustrated by the red arrows in Figure 3. To ease the analysis, we assume that the electron density variations, and therefore the local variations of the refractive index, are small within the size of the first Fresnel zone, which is in the order of $350 \mathrm{~m}$ for a typical L-band SAR. This allows us to use a simplified wave propagation model where we replace the wave equation and its forward-scattering solutions in terms of diffraction integrals by a geometric optics approximation which provides the range and phase offsets via a mere integration of the electron density along the ray paths [12]. We will later see that this approximation is well justified for the considered case of a moderately perturbed ionosphere where we predict notable interferometric phase errors but almost no amplitude scintillation in L-band. Using the geometric optics approach, the second-order correlation $C_{\varphi}\left(\theta_{1}, \theta_{2}\right)$ of the phase fluctuations of the single-pass SAR interferometer can be derived in case of small phase errors as

$$
\begin{aligned}
& C_{\varphi}\left(\theta_{1}, \theta_{2}\right)=E\left[\varphi\left(\theta_{1}\right) \cdot \varphi\left(\theta_{2}\right)\right]= \\
& \quad \iiint_{-\infty}^{\infty} H_{p}\left(f_{x}, f_{y}, f_{z} ; \theta_{1}, \theta_{2}\right) \cdot S_{N_{e}}\left(f_{x}, f_{y}, f_{z}\right) \cdot d f_{x} d f_{y} d f_{z}
\end{aligned}
$$

where $H_{p}\left(f_{x}, f_{y}, f_{z} ; \theta_{1}, \theta_{2}\right)$ is given by

$$
\begin{gathered}
H_{p}\left(f_{x}, f_{y}, f_{z} ; \theta_{1}, \theta_{2}\right)=\frac{\lambda^{2} r_{e}^{2}}{h_{i}^{2} \cos \theta_{1} \cos \theta_{2}} \iint_{-\infty}^{\infty} g\left(z^{\prime}\right) g\left(z^{\prime \prime}\right) . \\
\iint_{-\infty}^{\infty} h\left(\xi^{\prime}\right) h\left(\xi^{\prime \prime}\right) \exp \left[j 2 \pi f_{y}\left(z^{\prime} \tan \xi^{\prime}-z^{\prime \prime} \tan \xi^{\prime \prime}\right)\right] d \xi^{\prime} d \xi^{\prime \prime} . \\
\quad \exp \left[j 2 \pi\left(f_{x}\left(z^{\prime} \tan \theta_{1}-z^{\prime \prime} \tan \theta_{2}\right)+f_{z}\left(z^{\prime \prime}-z^{\prime}\right)\right)\right] d z^{\prime} d z^{\prime \prime}
\end{gathered}
$$

Here, $\lambda$ denotes the wavelength, $r_{e}$ the classical electron radius, $\theta_{1}$ and $\theta_{2}$ the slightly different incident angles of the two SAR channels, $\xi$ the instantaneous squint/azimuth angle, $h(\xi)$ the combined azimuth weighting from the antenna pattern and the SAR processing filter, and $h_{i}$ a normalization constant that is given by the integral of $h(\xi)$ over all $\xi$. The phase correlation can therefore be derived from a weighted 3-D projection of the power spectral density $S_{N_{e}}\left(f_{x}, f_{y}, f_{z}\right)$. To get a first idea of how higherorder TEC variations may impact single-pass cross-track interferometry, we model the three-dimensional electron density spectrum by an isotropic power law model [13]

$$
S_{N_{e}}\left(f_{x}, f_{y}, f_{z}\right)=\frac{A}{\left(f_{o}^{2}+f_{x}^{2}+f_{y}^{2}+f_{z}^{2}\right)^{\frac{\alpha}{2}}}
$$

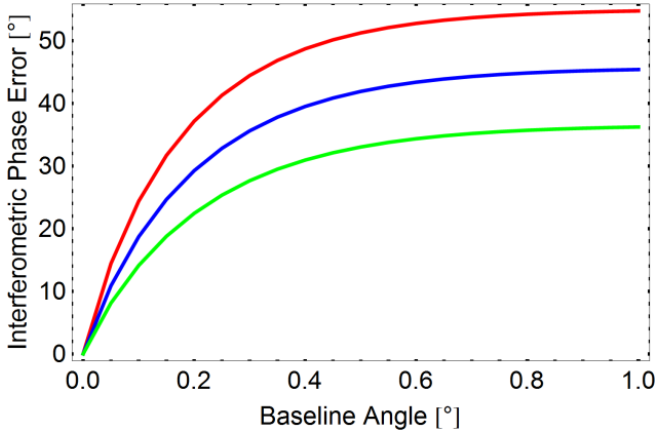

Figure 4: Predicted interferometric phase errors of an L-band single-pass SAR interferometer for a turbulent ionosphere. The total electron content of the background ionosphere is assumed as 40 TECU and the percentage of the variable content is $2 \%$. The spectral index and outer scale length are $\alpha=3.5$ and $10 \mathrm{~km}$, respectively. A Gaussian profile $g(z)$ has been used with a ionospheric height of $350 \mathrm{~km}$ and a thickness of $100 \mathrm{~km}$. The incident angle is $\theta_{i}=45^{\circ}$ and the synthetic aperture angular intervals are $\xi_{b}=2 \cdot 10^{-2}$ (green), $\xi_{b}=1 \cdot 10^{-2}$ (blue) and $\xi_{b}=1 \cdot 10^{-6}$ rad (red), where the first two values correspond for an L-band SAR to azimuth resolutions in the order of $6 \mathrm{~m}$ and $12 \mathrm{~m}$. The last $\xi_{b}$-value can be understood as the single pulse phase error for a point scatterer. Baseline angles of $\Delta \theta=0.1^{\circ}$ and $\Delta \theta=1.0^{\circ}$ correspond to a height of ambiguity of $100 \mathrm{~m}$ and $10 \mathrm{~m}$, respectively. The size of the first Fresnel zone corresponds in this example to a baseline angle of $\Delta \theta=0.04^{\circ}$ and the associated phase error is below $15^{\circ}$. Hence, no notable amplitude scintillation has to be expected for the individual radar pulses in this example.

where $\alpha$ denotes the 3-D spectral index and $f_{o}$ limits the otherwise unlimited increase of the power spectrum for spatial frequencies near the origin. The parameter $1 / f_{o}$ is known as the outer scale length which is often assumed to be in the order of $10 \mathrm{~km}$ [4]. The model can be easily extended to describe, for example, magnetic field aligned anisotropies in the Equatorial region by an appropriate frequency scaling and a subsequent coordinate rotation [14].

Using this correlation model, we can derive the interferometric phase errors via the phase structure function

$\overline{\Delta \varphi\left(\theta_{1}, \theta_{2}\right)^{2}}=C_{\varphi}\left(\theta_{1}, \theta_{1}\right)+C_{\varphi}\left(\theta_{2}, \theta_{2}\right)-2 C_{\varphi}\left(\theta_{1}, \theta_{2}\right)$ (8)

Figure 4 shows an example of the predicted standard deviation of the interferometric phase errors assuming the parameters provided in the figure caption. It becomes clear that already moderate levels of ionospheric disturbance can cause significant interferometric phase errors (the chosen parameters correspond to a vertically integrated strength of turbulence of $C_{k} L=6.5 \cdot 10^{33}$ ). It is important to note that these interferometric phase errors will not be reduced by multi-looking, since they are highly correlated among neighboring resolution cells. Irregularities in the ionosphere may therefore pose a potential challenge for both singlebaseline DEM generation as well as multi-baseline SAR interferometry and tomography that combine the data from multiple single-pass acquisitions. The same applies to multistatic SAR systems that acquire multi-baseline data in a single pass of the satellite formation [15]. 


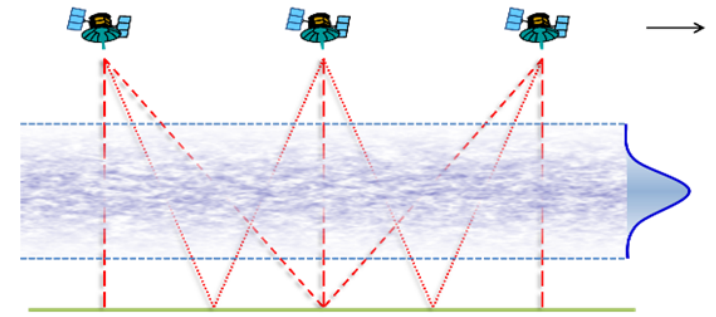

Figure 5: Satellite formations can acquire redundant SAR images that are well suited to analyze higher-order perturbations in the ionosphere.

\section{DISCUSSION}

We have shown that ionospheric TEC gradients and higherorder plasma irregularities may have a notable impact on single-pass SAR interferometry and tomography. These effects should therefore be taken into account when designing low-frequency bistatic and multistatic SAR missions and their associated signal processing chains. For this, a systematic study should be conducted that predicts the diurnal, seasonal and geographic distributions of the expected ionospheric phase errors based, e.g., on existing climatological scintillation models like WBMOD. This information can then provide valuable inputs to optimize the orbit, the satellite formation, and the data acquisition plan. Further, new processing techniques should be developed that allow for a mitigation of the phase errors. The processing may exploit the opposite signs of the radargrammetric and interferometric offsets in Equation (3) or the related $\Delta k$ technique, the processing of multiple sublooks in azimuth, and/or estimates of Faraday rotation in a fully polarimetric SAR interferometer. Further opportunities arise from the combination of single and repeat-pass interferometric SAR data.

Besides these challenges, single-pass interferometry and tomography offer also new opportunities to investigate the ionosphere and its impact on wave propagation. One example is the examination of small-scale electron density perturbations by a satellite formation employing two or more platforms that are mutually displaced in the alongtrack direction [15], [16]. Figure 5 illustrates such a SAR train for the case of three fully active radar satellites that may be operated in alternating bistatic or a more advanced MIMO-SAR mode [17]. Since each satellite can transmit and receive, we obtain in total 3 monostatic and 6 bistatic SAR images ( 3 bistatic images are, however, expected to be, within the start-stop approximation, redundant due to reciprocity). If we further assume that the scene is stationary and all images are processed within the same Doppler band, we may, for small bistatic angles, adopt the monostaticbistatic equivalence principle. We would, therefore, expect that all acquired mono- and bistatic SAR images are, up to mutual time shifts, equivalent. Deviations between the images could, however, occur due to the fact that the rays pass through different portions of the ionosphere. For this, one should note that spatial inhomogeneities in the ionosphere are often considered as temporally constant (or "frozen"), while the whole irregularity pattern may drift with velocities of several hundreds of meters per second, preferably in zonal directions [18]. A systematic evaluation of the mutual offsets in range, phase, amplitude and polarization of the otherwise redundant mono- and bistatic SAR images can therefore test this Taylor hypothesis of a frozen irregularity pattern and provide information about fine-scale structures at resolutions that are in the order of the Fresnel zone extent. As opposed to GPS measurements or dedicated experiments with beacon satellites, these data are not only available for single points, but simultaneously on a large plane that covers the whole swath imaged by the SAR.

\section{REFERENCES}

[1] G. Krieger, F. De Zan, M. Bachmann, P. Lopez Dekker, M. Rodriguez Cassola, JS. Kim, "Tropospheric and Ionospheric Effects in Spaceborne Single-Pass SAR Interferometry and Radargrammetry", European Conf. on Synthetic Aperture Radar (EUSAR), Berlin, pp. 1097-1100, 2014.

[2] G. Krieger, F. De Zan, M. Bachmann, J. Hueso Gonzalez, M. Rodriguez Cassola, M. Zink, "Unexpeted Height Offsets in TanDEM-X: Explanation and Correction", IEEE Geoscience and Remote Sensing Symposium (IGARSS), Munich, pp. 295-298, 2012.

[3] Z.W. Xu, J. Wu, Z.-S. Wu, "A Survey of Ionospheric Effects on SpaceBased Radar", Waves in Random Media, Vol. 14, pp.189-273, 2004

[4] D.P. Belcher and N.C. Rogers, "Theory and Simulation of Ionospheric Effects on Synthetic Aperture Radar", IET Radar, Sonar and Navigation, Vol. 3, No. 5, pp. 541-551, 2009.

[5] C.S. Carrano, K.M. Groves, and R.G. Gaton, "Simulating the Impacts of Ionospheric Scintiallation in L-Band SAR Image Formation", Radio Science, Vol. 47, RS0L20, 2012.

[6] F. Meyer, P. Rosen, X. Pi, K. Chotoo, K. Papathanassiou, JS. Kim, C. Carrano, "SAR and the Ionosphere: Challenges and Opportunities", UNAVCO Science Workshop, Broomfield, Colorado, 2014.

[7] H.B. Vo and J.C. Foster, "A Quantitative Study of Ionospheric Density Gradients at Midlatitudes", Journal of Geophysical Research, Vol. 106, No. A10, pp. 21555-21563, 2001.

[8] S. Pullen, Y.S. Park and P. Enge, "Impact and Mitigation of Ionospheric Anomalies on Ground-Based Aufgmentation of GNSS", Radio Science, Vol. 44, RS0A21, doi:10.1029/2008RS004084, 2009.

[9] K.C. Yeh and C.-H. Liu, "Radio Wave Scintillations in the Ionosphere", Proceedings of the IEEE, Vol. 70, No. 4, pp. 324-360, 1982.

[10] J. Aarons, "Global Morphology of Ionospheric Scintillation", Proceedings of the IEEE, Vol. 70, No. 4, pp. 360-378, 1982.

[11] A.W. Wernik, L. Alfonsi and M. Materassi, "Scintillation Modeling Using In Situ Data", Radio Science, Vol. 42, RS1002, 2007.

[12] A.D. Wheelon, "Electromagnetic Scintillation, I. Geometric Optics", Cambridge University Press, Boulder, USA, 2004.

[13] C.L. Rino, "A Power Law Phase Screen Model for Ionospheric Scintillation - 1. Weak Scatter", Radio Science, Vol. 14, pp. 1135-1145, 1979.

[14] C.L. Rino and E.J. Fremouw, "The Angle Dependence of Singly Scattered Wavefields", Journal of Atmospheric and Terrestrial Physics, Vol. 39, pp. 859-868, 1977.

[15] G. Krieger and A. Moreira, "Multistatic SAR satellite formations: Potentials and challenges", In: Proc. IEEE Geoscience and Remote Sensing Symposium (IGARSS), Seoul, Korea, pp. 2680-2684, 2005.

[16] A. Monti Guranieri, A. Broquetas, A. Recchia, F. Rocca, and J. Ruiz Rodon, "Advanced Radar Geosynchronous Observation System: ARGOS", IEEE Geoscience and Remote Sensing Letters, Vol. 12, No. 7, pp. 1406-1410, 2015.

[17] G. Krieger, "MIMO-SAR Opportunities and Pitfalls", IEEE Transactions on Geoscience and Remote Sensing, Vol. 52, pp. 2628-2645, 2014.

[18] A. Hewish, "The Diffraction of Galactic Radio Waves as a Method of Investigating the Irregular Structure of the Ionosphere", Proceedings of the Royal Society, Vol. 214, pp. 494-515, 1952. 